\title{
Cognitive Engagement: a More Reliable Proxy for Learning?
}

\author{
James D. Pickering ${ }^{1}$ (C)
}

Published online: 19 July 2017

(C) International Association of Medical Science Educators 2017

Student engagement is a topic of great discussion across all areas of higher education due to its obvious and potential links to assessment outcomes. Medical education is no exception, with conversations regularly held between faculty members to share best practice on how to promote student engagement. Recently, these conversations have focused on approaches that encourage attendance at teaching sessions and strategies to support the completion of work both before and after class. This focus has come to the fore as medical schools are now routinely utilising sophisticated tracking tools to assess attendance at timetabled teaching sessions and the number of times online materials are accessed. Personal experience highlights that this data can, to some extent, be a very useful mechanism to monitor student engagement and predict assessment outcomes. It is not surprising to see students who regularly miss teaching sessions or fail to access any of the teaching resources provided perform poorly. However, is this data driven - or behavioural-approach to student engagement the most reliable predictor of assessment outcomes?

Student engagement can be considered as the amount of time, energy and resource, devoted to activities designed to enhance learning [1]. Considerable work has been undertaken to characterise the underlying dimensions of engagement, with behavioural, emotional and cognitive all being identified. However, are these dimensions a reliable proxy for learning? If a student is emotionally, behaviourally and cognitively engaged, will they necessarily perform better in their assess-

James D. Pickering

j.d.pickering@leeds.ac.uk

1 Division of Anatomy, Leeds Institute of Medical Education, School of Medicine, University of Leeds, 9.06 Worsley Building, Clarendon Way, Leeds LS2 9NL, UK ment? Or, is an individual aspect of engagement a more reliable predictor for assessment outcomes - a more reliable proxy for learning? For example, integrating technologyenhanced learning resources into medical curricula and then collating the access data - behavioural engagement - may reveal a correlation with high assessment outcomes and therefore effective learning, but is this relationship causative? Using an example from my own teaching has revealed that those students, who scored the highest module marks, do not necessarily engage heavily with post-lecture recordings. Obviously, this example only focuses on one specific element of the course, and those students are likely to engage heavily with other resources, such as reading material or watching other videos, but that really is the point. The behavioural data we collect to make such judgements is limited to the behavioural data we collect. Clearly, these students were accessing other resources to achieve the learning objectives and pass the module, but this behaviour was out of our field of viewinvisible to our tracking system.

Similar conclusions can be drawn in regard to emotional engagement. Whereas students who smile, laugh and exhibit a sense of interest during class would be described as being engaged emotionally; others who attend class quietly and on the surface appear disinterested would be viewed as unengaged. However, far too often to be an exception, it is these former students who, despite appearing to be engaged emotionally, attend feedback sessions because they failed their assessment. In fact, during these face-to-face follow-up conversations and accessing their attendance data, it is obvious they were engaged both behaviourally and emotionally. Again, there will be exceptions to this, and those who constantly ask questions, attend all classes and interact with the tutors are likely to perform well, but having taught thousands of students, too many perform poorly despite their emotional and behavioural engagement data suggesting otherwise. 
The temptation to amalgamate the easily observable patterns of engagement - behavioural and emotional — with actual learning has been described by Mayer (2004) as the constructivist teaching fallacy [2]. Simply, engagement is a poor proxy for learning, and students need to do more than just appear to be physically interacting and enjoying their teaching sessions. Apart from setting a test or assessment, effective learning is difficult to visualise, and in order to provide maximal support to students, we need to find a way where our measure of engagement is causative. Having mentioned both behavioural and emotional engagement as useful predictors for assessment outcomes, the final domain of engagement to assess is cognitive. A cognitively engaged student would be one who is invested in their learning, embraces the challenge of acquiring new knowledge or skill sets, and goes beyond the course requirements. A tangible outcome for a student who is cognitively engaged would be to understand fully the learning objectives required for their course and be aware of the range of resources available to help them achieve these objectives. From this position, the student is able to make informed decisions on what needs to be learnt and the most appropriate ways to go about learning the material. It could be postulated, therefore, that it is cognitive, rather than emotional and behavioural engagement, that is the key domain in supporting effective learning. Recent work integrating social media as a platform for students to communicate with fellow students and the course leader revealed an interesting view of cognitive engagement [3]. Firstly, there was a group of active users who would pose questions and want to have various aspects of the curriculum explained or clarified; and secondly, another group of students who learned by reading the questions posted that a specific area of content was in fact a required component of the course. This range of cognitive engagement can be summarised as follows, and draws on terminology first termed by the US Secretary of Defence, Donald Rumsfeld in 2002 [4]. Some students clearly had a full awareness of the curriculum and were asking questions based on this contentthese students can be described as having known unknownsthat is, they know what they do not know. In addition, other students reported that by seeing the questions posed by their peers, they were made aware of curriculum content that they did not know needed to be known. These students can be described as having unknown unknowns - that is, they do not know what they do not know. This description of curricular engagement can be described along two axes: (1) an awareness of the required curriculum content, and (2) knowledge of that curriculum content (Fig. 1). Describing students with this range of curriculum engagement levels is a dynamic process as all students start with a series of unknown unknowns (or unknown knowns if the student has some preexisting knowledge from a previous educational experience), and by interacting with the curriculum, these hopefully become known unknowns. Once the student becomes aware of

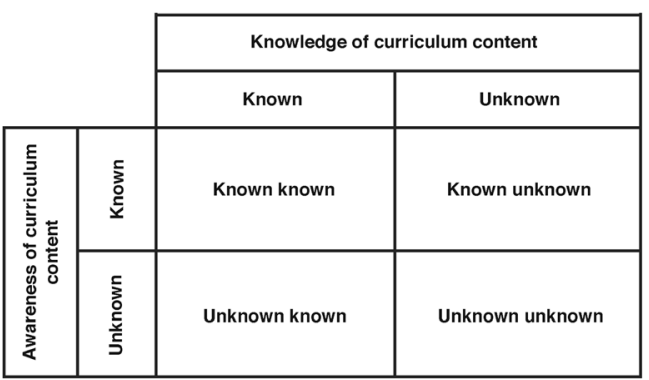

Fig. 1 Descriptions of curriculum engagement

their individual known unknowns, they can meaningfully participate with the specific learning opportunities (i.e. attending class, accessing online material or asking pertinent questions). The ultimate goal is to convert these known unknowns into known knowns - that is, being fully aware of the curriculum breadth and also knowledgeable of that content - and thereby achieve good assessment outcomes. Students who enter the assessment with enduring known unknowns or unknown unknowns are likely to perform poorly.

In this context, it could be suggested that cognitive engagement is a more reliable proxy for learning compared to either behavioural or emotional. However, cognitive engagement does not work in isolation and is merely the first step along the path to achieving successful learning outcomes. Each student must take personal responsibility to engage cognitively with their curriculum, and then take active steps to engage behaviourally, based on the increased understanding of their known knowns and known unknowns. The current work on learning analytics can provide additional and valuable support in regards to behavioural engagement and can lead to essential scaffolding and sign-posting to support students in making the best decisions in terms of their behaviour [5]. But, first of all, they need to be aware of the curriculum that surrounds themto engage cognitively. An essential endeavour for medical education teachers, therefore, is to ensure that each student is provided opportunities to immerse themselves in their course, and perhaps most importantly, explain why it is essential for their future medical careers that they understand this specific content. This is particularly important moving forwards as admissions into medical schools are increasingly becoming diverse, with each student having their own unique motivations, interests and drivers to learn specific areas of the curriculum. These inherent and personal motivations can sometimes clash with the robust and immobile curriculum components, such as timings and formats of teaching sessions. Nevertheless, despite these individual motivations, a vocational programme such as medicine, requires all students to be competent in a wide set of core components. Although it would be ideal for students to enjoy each and every teaching session or learning objective, this is not possible nor realistic, and therefore, ensuring students are knowledgeable of the 
content and how best to acquire that content is of paramount importance.

\section{Compliance with Ethical Standards}

Conflict of Interest The author declares that he has no conflict of interest.

\section{References}

1. Krause KL, Coates H. Students'engagement in first-year university. Assess Eval High Educ. 2008;33:493-505.
2. Mayer RE. Should there be a three-strikes rule against pure discovery learning? The case for guided methods of instruction. Am Psychol. 2004;59:14-9.

3. Pickering JD, Bickerdike SR. Medical student use of Facebook to support preparation for anatomy assessments. Anat Sci Educ. 2017;10(3):205-14.

4. Rumsfeld DH. Myers, R. Department of Defense: DoD News Briefing. U.S; 2002. http://archive.defense.gov/Transcripts/ Transcript.aspx? TranscriptID=2636. Accessed 13 July 2017

5. Saqr M, Fors U, Tedre M. How learning analytics can early predict under-achieving students in a blended medical education course. Med Teach. 2017; doi:10.1080/0142159X.2017.1309376. 